\title{
A tolerant approach
}

\section{Despite a long record of failure, a few immunologists continue to pursue precisely targeted therapies for autoimmune diseases.}

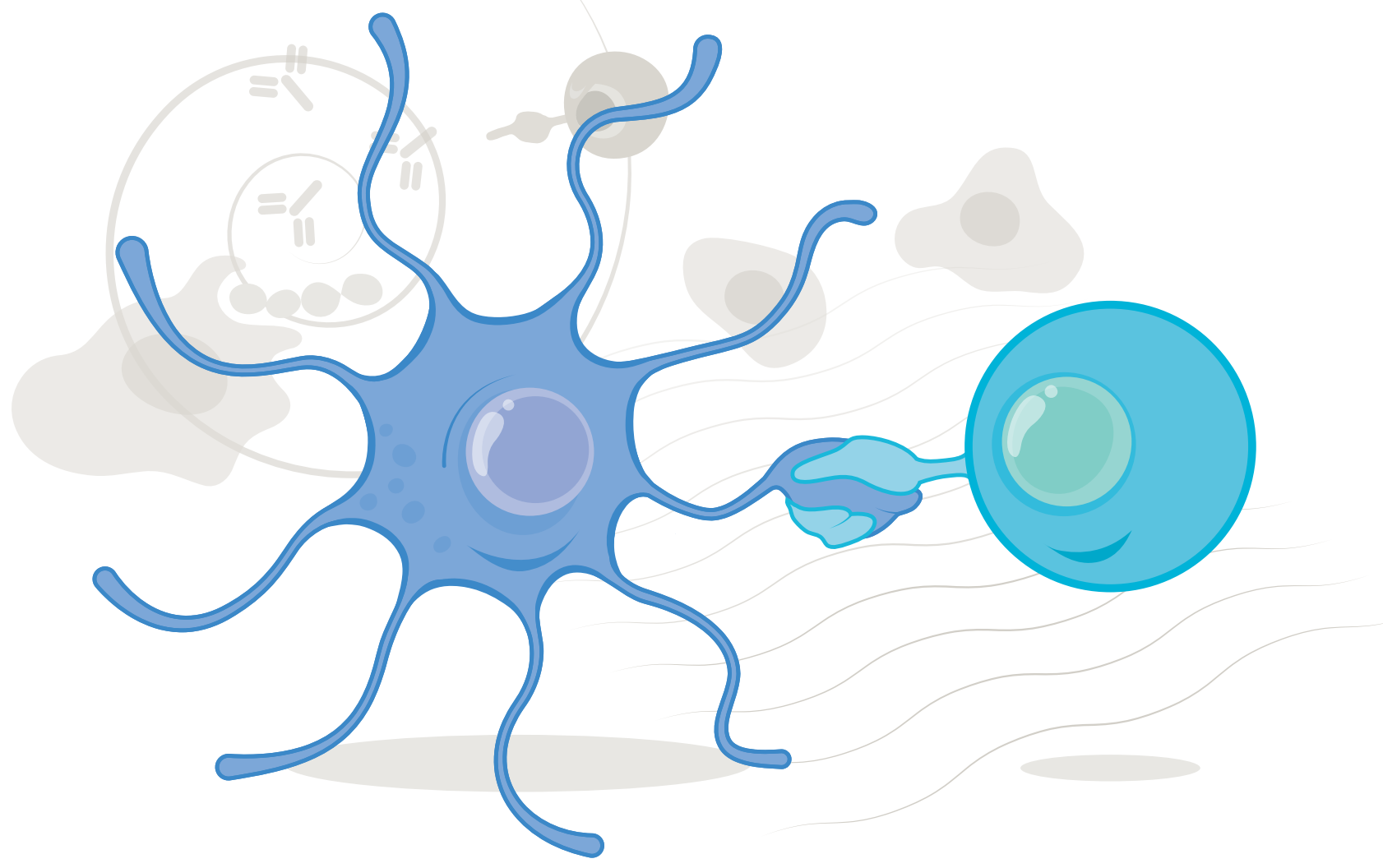

BY KEN GARBER

E ver since Ed Wiley learned that he had type 1 diabetes in 1997 , he has fretted over his meals, blood glucose levels and the daily programming of his insulin pump. Wiley, a statistician who lives outside Boulder, Colorado, and works on big data analytics, has learned to live in a state of hypervigilance. Finding the right dose of insulin turned out to be more art than science and, like many with the disease, his control began slipping away with time. By 2008, he says, "my insulin doses just basically didn't work any more". Unable to reliably anticipate what he needed, Wiley was having severe hypoglycaemic episodes and was at risk of diabetic seizures and long-term disability.

On his endocrinologist's advice, he enrolled in a clinical trial of a novel drug called BHT-3021. Although technically a vaccine, BHT-3021 is not designed to stimulate an immune response, but rather to shut it down, stopping the body's errant attack against cells in the pancreas that produce insulin. The goal is to achieve immune tolerance.

Drugs that broadly suppress immunity are the standard treatment for autoimmune disorders such as multiple sclerosis (MS), rheumatoid arthritis and lupus. But these drugs can lead to life-threatening 
infections, and do not address the cause of the disease. Tolerance therapies are different. They aim to target only the immune cells that react to a specific antigen, a substance - in Wiley's case, the insulin precursor proinsulin — that might trigger a response. "Why shut down a major arm of the immune system, if we're just trying to restore tolerance to one antigen?" asks immunologist Larry Steinman of Stanford University in California, who developed BHT-3021.

This strategy, known as antigen-specific tolerance, is simple in concept. But, so far, dozens of clinical trials have failed to achieve a categorical success. And there is a fine line between calming the immune system and stimulating it, so these efforts risk making a disease worse - as happened in an MS trial some 15 years ago.

BHT-3021 is one of a new wave of treatments conceived by five veterans of the field that promises to do better. Early-stage trials show encouraging results in people with MS and type 1 diabetes. "A number of these approaches really are going to work," predicts David Wraith, an immunologist at the University of Bristol, UK, and one of the few persisting in pursuing the work. "The science has caught up."

\section{AUTOIMMUNE OVERRIDE}

The approaches are varied, but they all rely on the body's natural ability to distinguish its own substances from those of foreign intruders. When bacteria or viruses invade, some are swallowed by specialized antigen-presenting cells, or APCs. These chop up the bacterial or viral antigens and present them to T cells, white blood cells that orchestrate the immune response. The T cells then proliferate and launch a coordinated attack.

APCs also ensure that normal daily maintenance does not turn deadly. As the body's own cells continuously die and are replenished, APCs mop up the debris and present those self-antigens to T cells along with an array of proteins that signal that these cellular remnants pose no danger. In autoimmunity, for unknown reasons, this protective mechanism goes awry. The new therapies are designed to override this dysfunction by deliberately sending the relevant antigen to tissues where the body is likely to see it as a non-threatening part of itself (see 'Teaching tolerance').

Most of the therapies developed so far target MS, which occurs when the immune system attacks the myelin sheath that protects neurons in the brain and spinal cord. Immunologist Stephen Miller of Northwestern University in Chicago, Illinois, designed a therapy ${ }^{1}$ that he and neurologist Roland Martin, now at University Hospital Zurich in Switzerland, began testing in patients in 2009. During the treatment, the patients' white blood cells are extracted, chemically linked to seven myelin antigens, then reinfused. The cells make their way to the spleen, where they die and release the antigen, which is picked up by APCs.

Wraith's drug ${ }^{2}$, ATX-MS-1467, uses four peptides, or pieces, of a myelin protein commonly attacked in MS. These injected antigens are taken up by immature APCs, which are incapable of stimulating T cells and instead inactivate them or convert them to a T-cell type that maintains tolerance. Krzysztof Selmaj's group at the Medical University of Lodz in Poland designed a similar therapy for the disease using three myelin peptides delivered through a patch that users wear on their skin ${ }^{3}$.

Instead of protein fragments, Steinman's diabetes treatment consists of circular pieces of DNA carrying the proinsulin gene, injected into mus$\mathrm{cle}^{4}$. The proinsulin protein is manufactured in the muscle cells, and is then secreted, taken up by APCs and presented to T cells. This produces "a signal that doesn't invoke danger but invokes tolerance", says Steinman. So far, this treatment and the other new therapies have been tested in fewer than 150 patients, but industry onlookers say they show promise.

\section{DANGEROUS GROUND}

The treatments not only have to overcome strong autoimmunity, they must also avoid making it worse. "We need to be extremely cautious," says Gerald Nepom, an immunologist at the Benaroya Research Institute in Seattle, Washington. Any novel manipulation of the immune system involves some risk. In 2006, an antibody-based treatment developed by the German drug company TeGenero was given to six healthy volunteers in a UK trial. Designed to quell the autoimmune response by a mechanism different from antigen-specific tolerance, it instead caused a massive immune response and multiple-organ failure ${ }^{5}$. The participants survived, and investigators have since implemented safer dosing protocols, but researchers know that an immune response can quickly go wrong. "People ask me what keeps me up at night," says Steinman. "Until we have quite a few patients under our belts for some time, it's

\section{"We have to take small steps and see what happens."}

\section{the worry that we're going to make things worse."}

Antigens can easily trigger immunity instead of tolerance, because there is a delicate balance between the two fates. Drug dose, delivery route, tissue destination and unpredictable changes in T-cell identity all matter. In a trial for an MS treatment that began in 1998 (ref. 6), doctors gave patients a single modified myelin peptide, but the trial was halted after three of the first eight recipients suffered worsening symptoms; one was left unable to walk. All three recovered with immunosuppressive treatment, but tests clearly implicated the experimental therapy, and the researchers ultimately worked out that they had extrapolated too high a dose from earlier in vitro studies. "We were both frustrated and also shocked," says Martin, a lead investigator on the trial.

Most trials so far have simply failed to work. In 2009, a trial of a myelin peptide antigen involving 612 people with MS showed no benefit over a placebo ${ }^{7}$. One likely reason is that the immune response in most autoimmune diseases can shift from one antigen to another as tissue damage progresses. Miller documented this phenomenon, known as epitope spreading, in animal models 20 years ago ${ }^{8}$. Martin points out that the failed 2009 trial used a single antigen, and adds that the patients involved were at a very advanced stage of the disease, when the immune system is no longer the main cause of neuronal damage.

Wraith says that the field has learned from its mistakes. The new therapies all incorporate multiple antigens to anticipate epitope spreading, for example. And researchers are paying close attention to other relevant factors, such as how the drugs are administered. In the past, says Christophe Benoist, an immunologist at Harvard Medical School in Boston, Massachusetts, it was often more of a gamble. The approach, he says, was, "Let's just put the antigen in and hope something good happens".

Richard Ransohoff, an MS researcher at the Cleveland Clinic in Ohio, has faith in the new therapies, which build on recent advances in understanding antigens and T cells. "These are all very experienced people who understand the complexity of what they're trying to do," he says. That is not to say that the mechanisms of tolerance have been completely worked out. "We're working furiously," says Steinman, but he admits that they are operating in "a vast ocean of ignorance in between icebergs of knowledge". Waiting for that perfect understanding, however, seems foolish. "We have to take small steps and see what happens," he says.

Results from the latest wave of trials are reassuring. Martin's clinical team gave nine patients a single injection of the manipulated immune cells, in escalating doses. The treatment seems to be safe, and the four patients receiving the highest doses showed a reduction in the number of T cells targeting self-antigens". "That was a very positive proof-ofconcept study," says Nepom, who was not involved in any of the trials.

The most promising trial, he adds, was a 30-patient test of Selmaj's therapy. Compared with a placebo, the treatment achieved a statistically significant drop in MS disease activity, as measured by magnetic resonance imaging of the brain. Patients assigned to the therapy also had far fewer relapses ${ }^{10}$.

In Wraith's ATX-MS-1467 trial, sponsored by Apitope in Diepenbeek, Belgium, 43 patients received the treatment in a series of five escalating 


\section{Teaching tolerance}

To stop the immune system from attacking healthy tissues, as in autoimmune disease, researchers are exploring ways to induce tolerance, essentially achieving the opposite goal of a conventional vaccine. They focus on how immune cells called $T$ cells are trained to recognize antigens - peptides and other molecules that the immune system can identify as a threat.

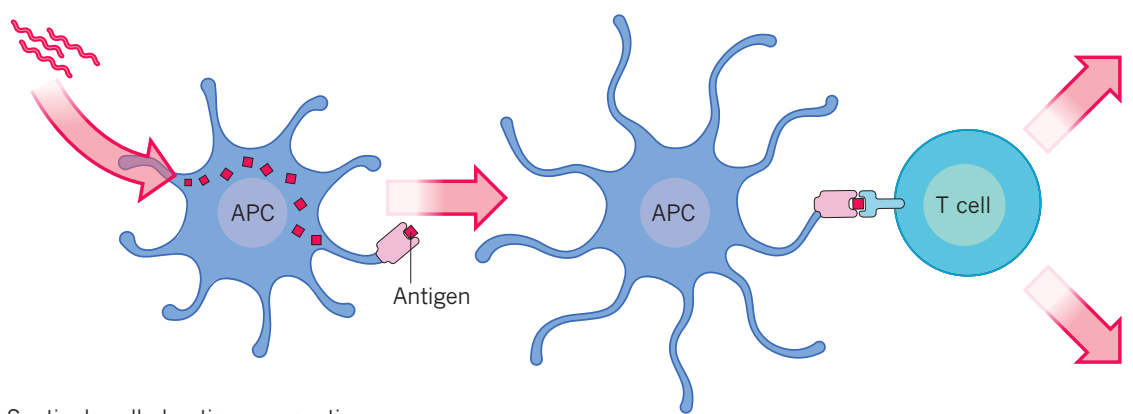

Sentinels called antigen-presenting cells (APCs) engulf and chop up biological molecules, and present them on specialized surface proteins.
A T cell has a receptor that binds to the presented antigen.

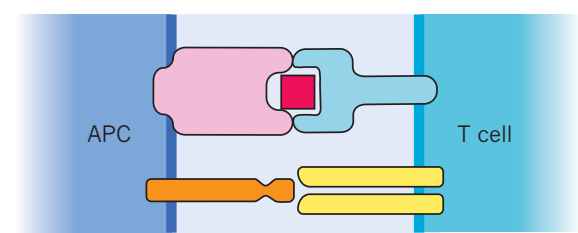

In conventional vaccination, the antigen generally appears along with other 'co-stimulatory' molecules on the surface of the APC. This combination puts the T cells on the alert and helps immunize against anything bearing that antigen.

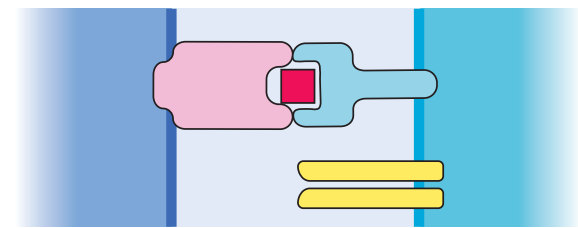

Without co-stimulation - or in the presence of other inhibitory signals - the T cell dies, becomes inactivated or takes on a regulatory role, preventing other immune cells from attacking healthy tissue. This is the goal in antigen-specific tolerance. doses either under or into the skin. Those in the latter group showed a large reduction in MS activity, says Wraith, although this returned three months after the treatment ended. The data have not yet been published.

Steinman's 80-patient, placebo-controlled, BHT-3021 diabetes trial established that the drug was safe, and one dosage group showed a statistically significant increase in a marker of insulin secretion and a simultaneous decrease in the number of T cells targeting proinsulin ${ }^{11}$.

Wiley believes the treatment has helped. During the 12-week study, he says, he regained control of his insulin dosing. And, subjectively at least, the positive effects have persisted.

\section{MOVING FORWARD}

But the legacy of failure has left many researchers - and drug companies - deeply sceptical. Even after positive trial results, Martin says, there are "very few investigators that are masochistic enough over a long time to continue in this field, because it's difficult to get funding". He and Selmaj are trying to get support for their new MS trials. And Steinman and the company he co-founded, Tolerion of Portola Valley, California, are seeking between US $\$ 20$ million and $\$ 30$ million to take their diabetes vaccine to the next stage. "It's impossible to say when this is going to happen," Steinman says. His first diabetes trial was sponsored by the biotech giant Genentech, based in South San Francisco, California. But after acquiring the company in 2009, Swiss drug firm Roche sought to get out of type 1 diabetes therapies, Steinman says. The company returned the therapy's licence even before the trial ended, and terminated all future commitments.

Others have had more success. Another Swiss drug company, Merck Serono, has already moved Wraith's therapy into phase II to confirm its efficacy. Miller, meanwhile, is planning a phase I trial of a variation on his original approach that uses biodegradable nanoparticles instead of blood cells as antigen carriers ${ }^{12}$. And Chicago-based COUR pharmaceuticals - co-founded by Miller - has signed partnerships with two drug companies for trials in people with diabetes and coeliac disease.

The next round of trials, Nepom says, should incorporate detailed mechanistic studies "in order to learn whether we've chosen the right antigen, and whether we've chosen the right dose and route" in treated patients. This is because, in diseases such as MS and diabetes, researchers can only guess which antigen triggers the immune response at any point in time. "That's an incredibly challenging problem," says Nepom.

He heads the Immune Tolerance Network (ITN), a US-led international consortium that allocates roughly $\$ 27$ million a year from the National Institutes of Health for clinical trials and related studies. In his view, antigen therapies should be used in combination with drugs from a second major category of tolerance treatment. Unlike antigen-specific therapies, these treatments do not inactivate, convert or destroy the relatively small number of $\mathrm{T}$ cells that attack a given antigen. Instead, they are designed to tilt the balance of the body's T-cell repertoire away from the subtypes that promote inflammation and towards those that maintain a state of tolerance, without impairing normal immunity against pathogens. These tolerance treatments have worked only temporarily, if at all, in autoimmunity trials. Nepom thinks that combining the two approaches will produce longer-lasting results. The ITN will soon require that all of the antigen trials it supports be combination trials.

"That's definitely the path to go," agrees Benoist. "Whether that will work or not, who knows. But at least that's a more rational way of doing things."

But pushing combinations does not sit well with some in the field. Miller, for example, doesn't think that the second approach achieves true tolerance, and he fears that combining it with antigen therapies will convolute the results. "If you want to test tolerance, it really has to be done as an individual entity," he says. Wraith thinks that adding the other class of treatment may even interfere with antigen-specific tolerance.

But the combination approach should reduce risk, say Nepom and ITN founder Jeff Bluestone, an immunologist at the University of California, San Francisco. This is because shifting the overall system towards tolerance should help to blunt any unexpected reaction to antigens.

Miller and Wraith say that their treatments have already proved safe in patients, and Wraith debated with Bluestone during a meeting in the Netherlands last October. Wraith calls Bluestone's concerns unfounded. Bluestone's reply: "I hope he's right."

Wiley, for his part, had no problems while taking BHT-3021. The only side effect he noticed was the welcome disappearance of some painful plantar warts. He thinks that BHT-3021 stabilized his body's ability to produce insulin, and would gladly take part in a longer study. "No question," he says. "I would jump at the opportunity."

Ken Garber is a science writer in Ann Arbor, Michigan.

1. Getts, D. R. et al. J. Immunol. 187, 2405-2417 (2011).

2. Gabryšová, L. et al. J. Exp. Med. 206, 1755-1767 (2009).

3. Juryńczyk, M. et al. Ann. Neurol. 68, 593-601 (2010).

4. Solvason, N. et al. J. Immunol. 181, 8298-8307 (2008).

5. Suntharalingam, G. et al. N. Engl. J. Med. 355, 1018-1028 (2006).

6. Bielekova, B. et al. Nature Med. 6, 1167-1175 (2000).

7. Freedman, M. S. et al. Neurology 77, 1551-1560 (2011).

8. McRae, B. L., Vanderlugt, C. L., Dal Canto, M. C. \& Miller, S. D. J. Exp. Med. 182, 75-85 (1995)

9. Lutterotti, A. et al. Sci. Transl. Med. 5, 188ra75 (2013).

10.Walczak, A., Siger, M., Ciach, A., Szczepanik, M. \& Selmaj, K. J. Am. Med. Assoc. Neurol. 70, 1105-1109 (2013).

11. Roep, B. O. et al. Sci. Transl. Med. 5, 191 ra82 (2013)

12.Getts, D. R. et al. Nature Biotechnol. 30, 1217-1224 (2012). 